WAPD $-T-2943$

Page 1

\title{
THERMAL HYDRAULICS OF
}

\section{STEAM GENERATOR SLUDGE}

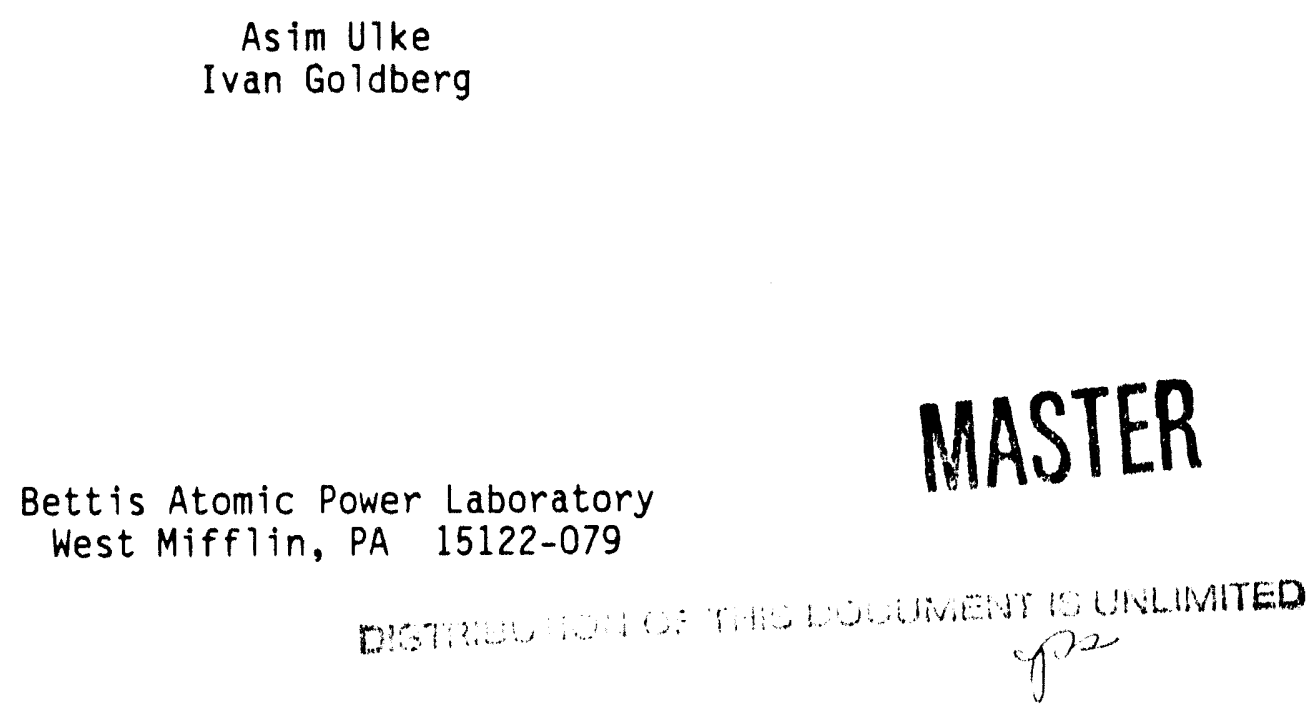

\section{DISCLAIMER}

This report was prepared as an account of work sponsored by an agency of the United States Government. Neituer the United States Government nor any agency thereof, nor any of their employees, makes any warranty, express or implied, or assumes any legal liability or responsibility for the accuracy, completeness, or usefulness of any information, apparatus, product, or process disclosed, or represents that its use would not infringe privately owned rights. Reference herein to any specific commercial product, process, or service by trade name, trademark, manufacturer, or otherwise does not necessarily constitute or imply its endorsement, recommendation, or favoring by the United States Government or any agency thereof. The views and opinions of authors expressed herein do not necessarily state or reflect those of the United States Government or any agency thereof. 
WAPD-T-2943

Page 2

Intentionally Blank 


\section{INTRODUCTION}

Experimental and analytical studies of thermal hydraulic processes in commercial steam generator tube sheet sludge have been previously reported ${ }^{1}{ }^{2}$. That work was performed because the authors believed that tubing corrosion occurs when the sludge deposit becomes too deep for the liquid to penetrate freely, leading to formation of a liquid deficient region with high chemical concentrations on the tube surface.

The primary objective of this work is to determine analytically the extent of liquid penetration into porous sludge. The secondary objectives are determinations of liquid saturation and chemical concentration profiles along the sludge covered tube length.

The method described in this paper differs from those used in previous works ${ }^{1}{ }^{2}$ in that it allows specification of porosity and permeability as a function of distance into the sludge and, also, in some of the auxiliary equations used.

\section{ANALYTICAL MODEL}

The model considers a slab of porous medium on a horizontal nonporous surface, both underwater. Heat is provided by a vertical tube. The vapor flows vertically upward and the liquid is replenished by a counterflow. The thermal hydraulics along the wetted length are described by five first order differential equations. The continuity for the liquid and the vapor are given by,

$$
\begin{aligned}
& \rho_{\ell} \frac{d V_{s \ell}}{d x}+\lambda_{\ell g}=0 \\
& \rho_{g} \frac{d V_{s g}}{d x}-\lambda_{\ell g}=0
\end{aligned}
$$


where $\lambda_{\ell g}$ is the vaporization rate per unit volume. $V_{s e}$ and $V_{s g}$ are the superficial velocities for the liquid and the gas phases. The densities for the liquid and the gas phases are represented by $\rho_{\ell}$ and $\rho_{g}$.

The velocities can be related to the pressure gradients by the Kozeny-Carman equation. ${ }^{3}$

$$
\begin{aligned}
& \frac{d P_{\ell}}{d x}=-\frac{\mu_{\ell}}{k_{r \ell} k} v_{s \ell}-\frac{\rho_{\ell}}{n_{r \ell} n} v_{s \ell}^{2}+\rho_{\ell} g \\
& \frac{d p_{g}}{d x}=-\frac{\mu_{g}}{k_{r g} k} v_{s g}-\frac{\rho_{g}}{n_{r g} n} v_{s g}^{2}+\rho_{g} g
\end{aligned}
$$

where $\mu_{\ell}$ and $\mu_{g}$ are viscosities, $K_{r \ell}$ and $K_{r g}$ are the relative laminar permeabilities, $n_{r l}$ and $n_{r g}$ are the relative turbulent permeabilities for the liquid and the vapor respectively, and $k$ is the laminar permeability given by,

$$
k=\frac{\varepsilon^{3} d_{p}^{2}}{150(1-\varepsilon)^{2}}
$$

and $\mathrm{n}$ is the turbulent permeability obtained from the Ergun's model."

$$
n=\frac{\varepsilon^{3} d_{p}}{1.75(1-\varepsilon)}
$$

The porosity is represented by $\varepsilon$ and the particle diameter by $d_{p}$.

The relative permeabilities, $K_{r \ell}$ and $K_{r g}$ were calculated using the forms developed by Verma, et. al. ${ }^{5}$ The turtulent relative permeabilities, $n_{r \ell}$ and $n_{r g}$ were assumed to have the same values as $k_{r l}$ and $k_{r g}$ respectively.

Leveret ${ }^{6}$, has shown that capillary pressure $P_{C}$ versus normalized liquid saturation $S$ can be correlated in the following form.

$$
f=1.417(1-S)-2.120(1-S)^{2}+1.263(1-S)^{3}
$$


where $f$ is the dimensionless pressure defined by,

$$
f=\frac{P_{C}}{\sigma}\left(\frac{k}{\varepsilon}\right)^{1 / 2}
$$

where $P_{C}$ is the difference of gas and vapor pressures, and $\sigma$ is the surface tension.

The evaporation rate can be calculated from

$$
\lambda_{\ell g}=\frac{1}{A h_{f g}} \frac{d \dot{q}}{d x}
$$

where $\mathrm{r}_{\mathrm{fg}}$ is the latent heat of vaporization.

The heat transfer rate $\dot{q}$ per unit length can be calculated in the usual manner by iterative techniques. Here the boliing heat transfer coefficient was calculated using Thom's correlation ${ }^{7}$, and adjusted by the porosity and the liquid saturation.

The concentration of chemical species can be obtained approximately from,

$$
\frac{d C}{d x}=C \lambda_{\ell g} / \rho_{\ell} V_{s \ell}
$$

The above first order differential equations can be solved by numerical integration as an initial value problem. The iritegration starts from the known values of pressure and liquid saturation with an assumed value of the liquid velocity at the top boundary and proceeds until either $v_{s e}$ or $S$ reach zero. At this point the initial value of the liquid velocity is adjusted using an iterative scheme to bring both the $V_{s l}$ and $S$ within convergence.

\section{RESULTS AND DISCUSSION}

Test results of liquid penetration into porous media were presented in Reference (1) and (2). The calculational procedure described here was applied 
WAPD-T-2943

Page 6

to these tests. A reasonable correlation to measured wetted lengths was obtained by using constant porosity and permeability for a set of tests where the porous bed maintained its integrity ${ }^{2}$. A second set of tests where vertical cracks $*$ re observed in the top section of the granular bed 'was also successfully represented by assigning a higher effective permeability to the top section. These results are compared to the test data in Figure 1. An interesting result of the two region representation of the sludge pile appears in the liquid saturation profile shown in figure 2. There is a region of increased liquid saturation between the layers of high and low permeability. The horizontal cracks observed in the bed ${ }^{1}$ may have been due to this phenomenon. Figure 3 shows the hypothetical chemical concentration profile. These results show that the cracks on top of a sludge pile supply the liquid necessary for deeper penetration into the sludge. By use of this method it is possible to identify tube locations within the sludge pile which are susceptible to corrosion, and thus, to provide guidance for planned nondestructive inspections. 
WAPD-T-2943

Page 7

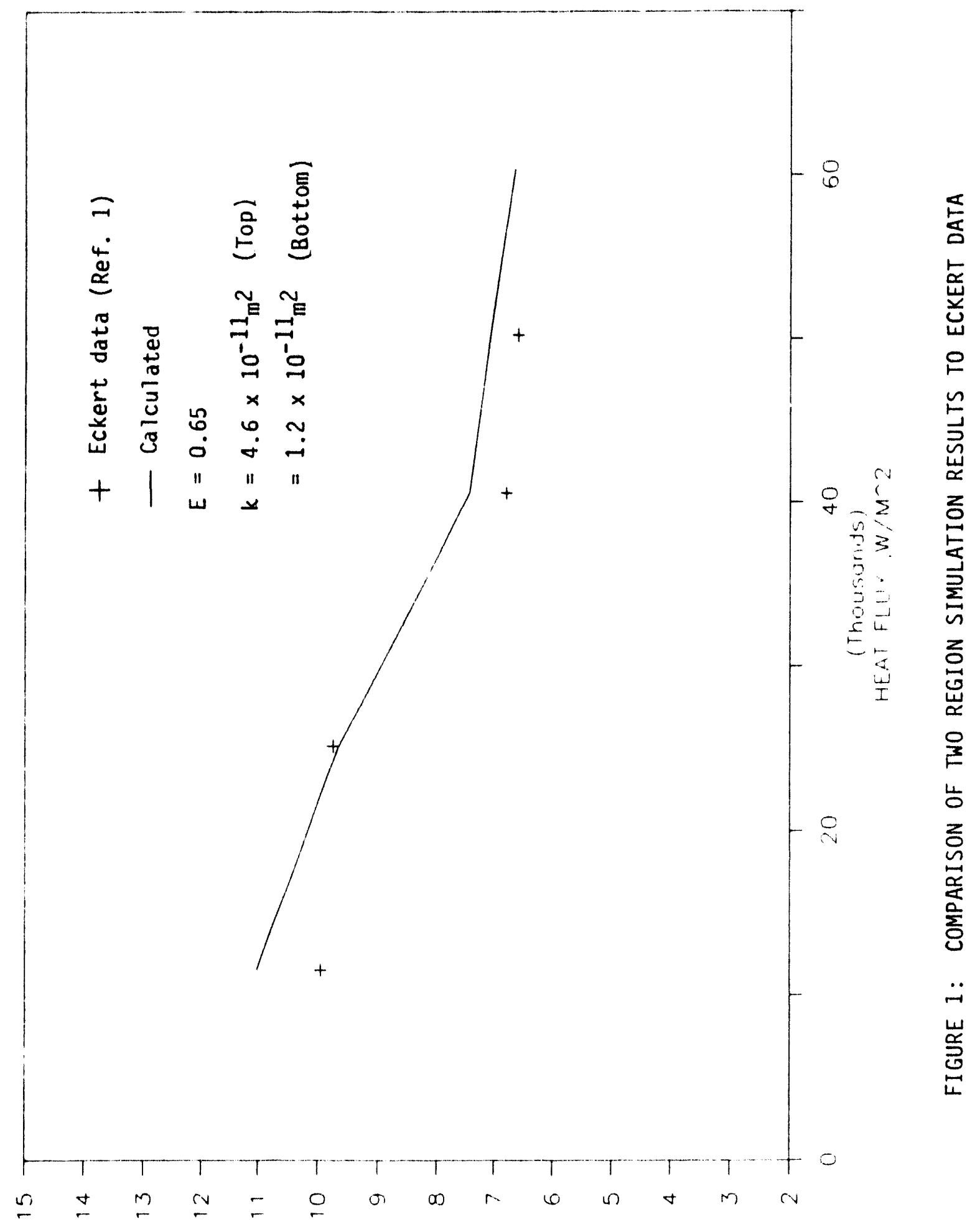




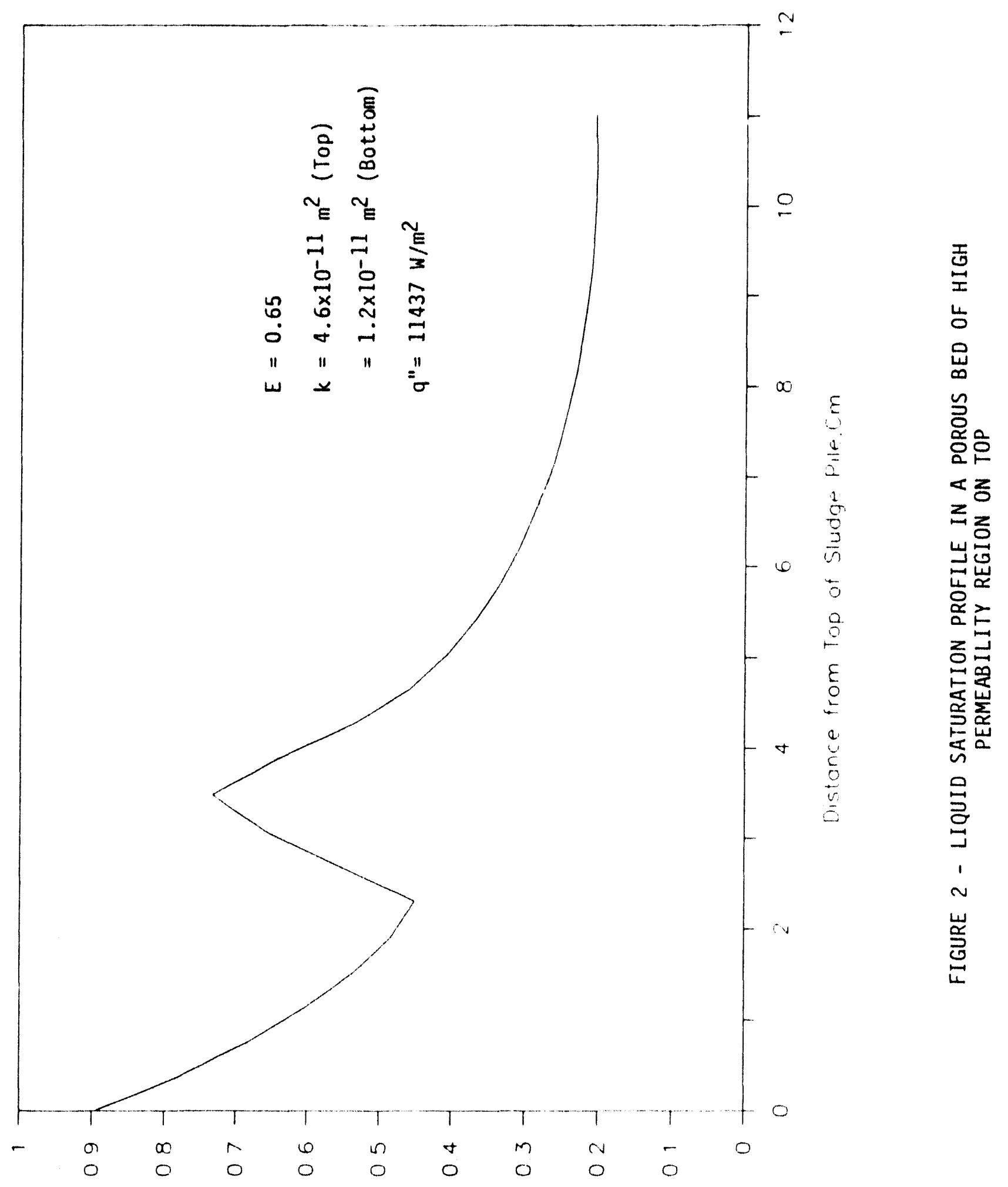




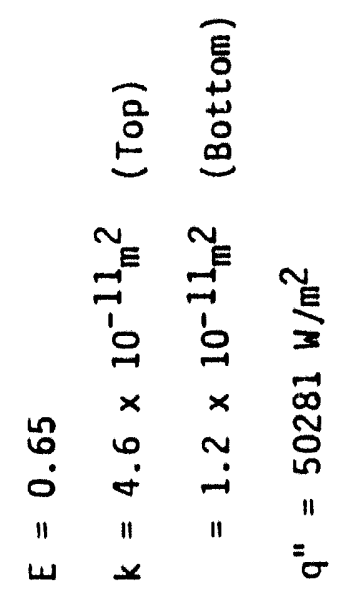


WAPD - T -2943

Page 10

\section{REFERENCES}

1. E.R.G. Eckert, et al., "A Study of the Bolling Processes in the Sludge Deposit of Steam Generators", EPRI NP-3018, May 1983

2. A. J. Baum and P. K. Greaney, "An Experimental and Analytical Investigation of Bolling Heat Transfer in Porous Bodies", ASME/AIChE N.H.T.C., Milwaukee, 1981, ASME Paper No. 81-HT-44 (EI 046670, 1982)

3. W. Chu, et a1., "Study of Pressure Drop, Vold Fraction and Relative Permeabilities of Two Phase Flow Through Porous Media". AIChE Symposium Series No. 225, Vol. 79, 1983, pp. 224-235

4. S. Ergun, "Fluid Flow Through Packed Columns", Chem. Engrg. Progress, V48 \#2, 89-94, February 1952 (EI 371, 1952)

5. A. K. Verma, et a1., "A Study of Two Phase Concurrent Flow of Steam and Water in an Unconsolidated Porous Medium", LBL-19084, DE-85014534, 11 pages (1985)

6. M. C. Leverett, "Capillary Behavior in Porous Solids", AIME Trans. Vol. 142, 1941, pp. 152-169

7. J.R.S. Thom, et al., "Boiling in Subcooled Water During Flow in Heated Tubes or Annuli", Proc. Inst. Mech. Engrs. Vol. 180, Part 3C, pp. 226-46, 1965-66 (EI 1126, 1967)

WP : SGA890459C 


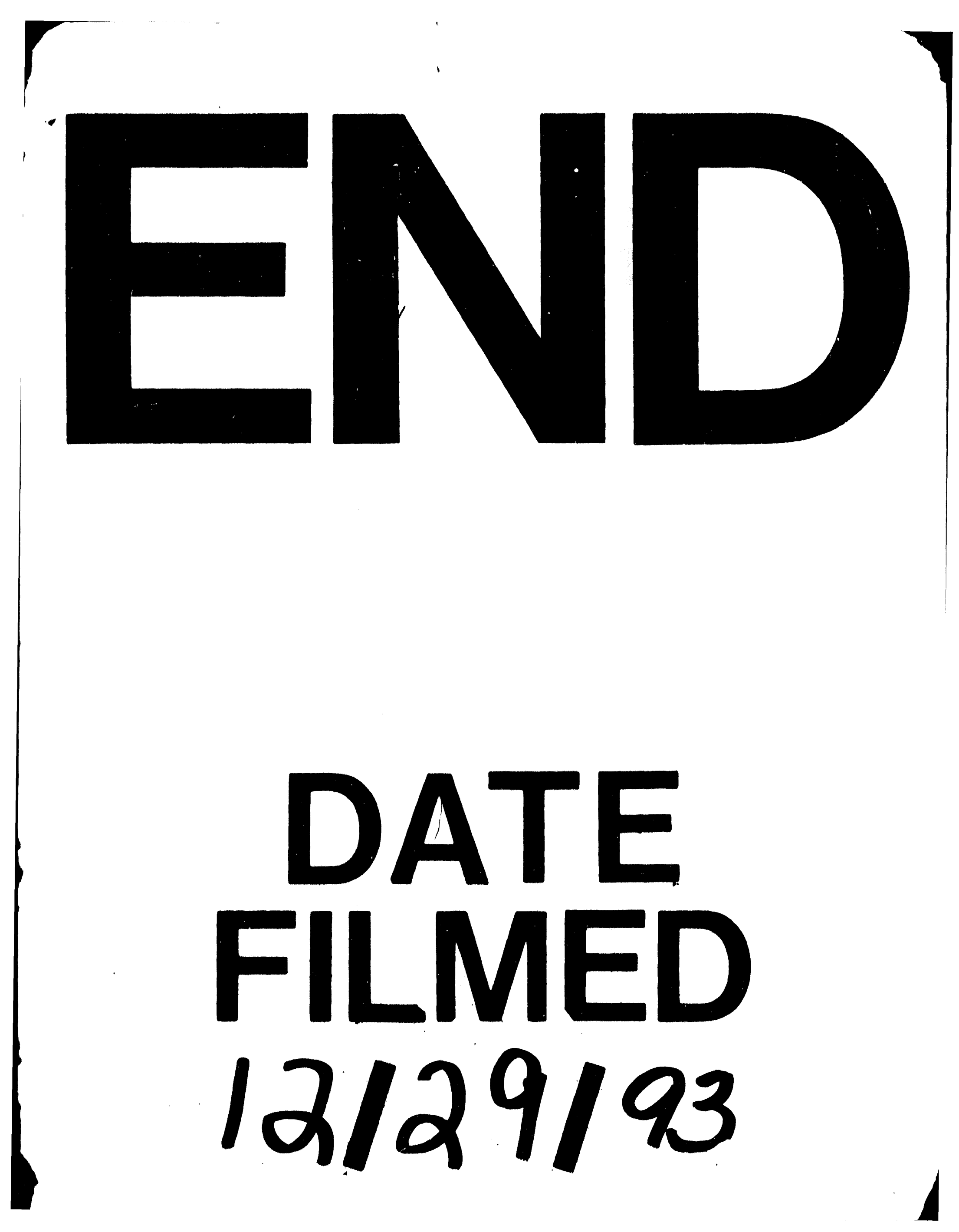


